\title{
Implementation of Cloud loT - Based Patient Fall Detection System with GeoTagging using Tilt Sensors and Texas Instruments' CC3200
}

\author{
K. Evangili Supriya and K. Raghavendra Rao* \\ Department of Physics, Embedded System Laboratory, Sri Krishnadevaraya University, Ananthapuramu - 515003, \\ Andhra Pradesh, India; evangilisupriya.k@gmail.com, kanchiraghavendrarao@gmail.com
}

\begin{abstract}
Objective: Coupled with an elderly population and extended life expectancy, disorder in the movement is one of the leading causes of functional disability. In this study we have designed an IoT-based Patient Fall Detection System (PFDS) using a low-cost sensor SW-520D, cloud computing technology and GeoTagging. Methods/Analysis: The position of the patient is detected by using tilt sensor which uses the rolling ball technology and is interfaced with the CC3200 microcontroller which is Wi-Fi on chip module using $802.11 \mathrm{~b} / \mathrm{g} / \mathrm{n}$ network protocol. The location of the patient can easily be tracked by GeoTagging technique. Findings: when the patient is tilted on the wheel chair/adjustable bed the tilt sensor detects the tilted motion and data is processed to the microcontroller and the data will be uploaded through Thing Speak cloud which is used for storing the real time values and is represented graphically. SIM 900A GSM module is used to send an alert if abnormality detected in the patient position and the location of the patient can easily be accessed using GeoTagging. Conclusion: In this study we have developed a cost-effective IoT-based PFDS that can access the data in real time through Thing Speak cloud and an alert signal is sent to the nursing attendant and hospital dashboard.
\end{abstract}

Keywords: GeoTagging, Internet of Things (IoT), Patient Fall Detection System, Tilt Sensor, CC3200

\section{Introduction}

Development in the fields of wireless sensor technology and smart phones have paved a new way to the wearable fall detection system. As there are many ongoing efforts that are taking place in the field of patient fall detection system [PFDS] especially on the wheel chair/adjustable bed, many technical hurdles are needed to be resolved. Health care systems need to move towards more scalable, optimal, cost-effective and affordable paths. Restructuring health systems focussing on wellness rather than illness and early detection of chronic diseases will be helpful for the patient's life. In addition, user may feel more comfort if there is a continuous time sense monitoring which leads optimal maintenance and can be supervised during chronic conditions when an event triggered ${ }^{1}$.
Various researchers are working to aid communication and mobility of older adults lying on adjustable beds and people with disabilities to increase their quality of life and to give scope to lead autonomous and independent life style so that they will have a great potential to integrate with social environment ${ }^{2}$.

Quadriplegics are persons who are unable to use their hands/legs to any of the extremities. The reason behind for that decreased motion will be birth effects, accidents, diseases or by age ${ }^{3}$. The patients with such severe disabilities have to exert some force in order to move their wheel chair/ adjustable bed and they cannot perform their daily activities on their own".

This problem can be overcome in such patients to certain extent by using different wearable medical devices. If the patient on the wheel chair/ adjustable bed suddenly

${ }^{*}$ Author for correspondence 
become unconscious and falls forward as shown in the Figure 1, then an immediate attention is needed to take place.

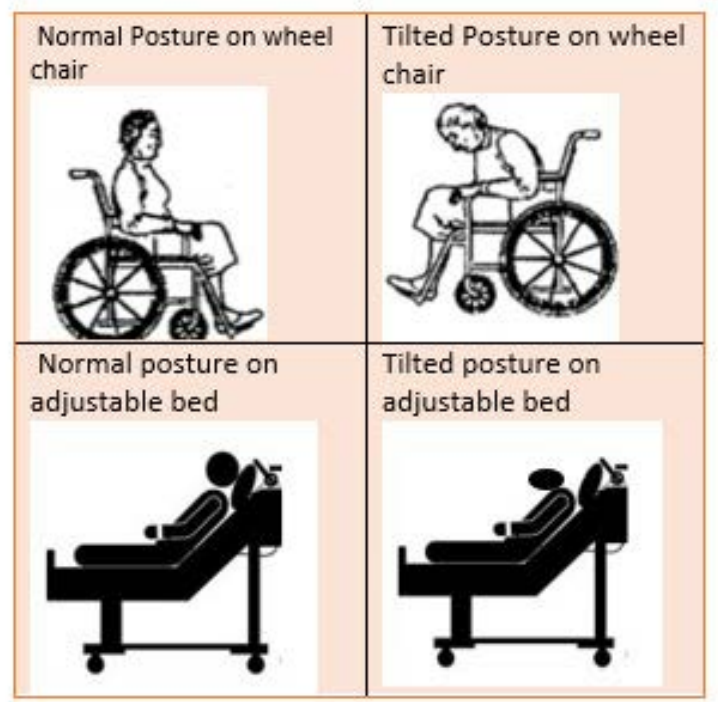

Figure 1. Normal and Tilted postures of patient on wheel chair/adjustable bed.

In such a scenario it can be handled by developing PFDS using Cloud Internet of Things (CIoT) and GeoTagging which can track the location of the patient easily. $\operatorname{In}^{5}$, have presented a review on wearable sensors to monitor older adults specially residing in rehabilitation centres. System review includes home monitoring and monitoring of patient's condition in clinics remotely and sending an alert when abnormality is detected. $\operatorname{In}^{6}$, a wheel chair communicator system was proposed by using tilt sensor and wireless module which can operate the direction of the wheel chair using head tilt movements especially by the people who are unable to move their hands or legs. $\mathrm{In}^{\mathrm{T}}$, have presented a prototype model for monitoring health parameters under Wireless Personal or Body Area Networks (WPANs and WBANs). In ${ }^{-}$, have developed an automated wheel chair which works by recognizing gestures of the head moments of the disabled person sitting on the wheel chair. In ${ }^{9}$ have implemented a wheel chair using accelerometer by hand gesture recognition. The direction of the chair will be sensed by accelerometer that detects the tilt and the corresponding signal is given to the microcontroller so that it controls the direction of the wheel chair. $\operatorname{In}^{\frac{10}{}}$, a smart wheel chair have developed for disabilities based on android control, where it consists of accelerometer which acts as a motion sensor control mechanism and even an alert will be sent to the care taker if the patient is in the critical condition. $\operatorname{In} \frac{11}{\text {, }}$ have developed a seating functions in usage of wheel chair environment where the functions include tilt-in-space, backrest position and seat elevation by using three tilt sensors. The study of time spent in different seating positions will enhance the better compliance of wheel chair position. $\operatorname{In} \frac{12}{2}$ dependent voice recognition automated wheel chair without manual interface was developed by integrating accelerometer sensor (ADXL335) and Ultrasonic sensor (HC-SR04) and Infrared sensor. In $\frac{13}{}$, have presented locomotion of wheel chair based on hand gesture wirelessly (RF modules) using triple axis accelerometer sensor (ADXL335), IR sensor and solar panel and the wheel chair will stop if it faces any obstacle. $\operatorname{In}^{14}$, have presented a MEMS technology based Wheel chair movement control using Mems accelerometer sensor ADXL335 which is capable of detecting tilt.

\subsection{Issues with the Existing Systems}

1. GeoTagging is not implemented.

2. Classification techniques involved are complex.

3. Time consuming and costly.

\section{Objectives of the Present Study}

It is clear that various researchers have used ADXL335 accelerometer sensor which leads to the complexity of the system and the technologies like RFID, Bluetooth, GPRS etc. for monitoring the patient position on the wheel chair and adjustable bed. Furthermore, an IoT Cloud based Patient Fall Detection System (PFDS) using tilt sensor SW-520D and GeoTagging is very rare. So, developing PFDS using a low-cost sensor SW-520D based on GeoTagging makes it really a bonus for the general public and the wireless system will give confidence and willpower to the patients on the wheel chair/adjustable bed. Keeping this in view point, we have designed an IoT based PFDS using SW-520D tilt sensor, Texas Instruments CC3200 and GSM module for wireless communication that includes Geo Tagging. The developed system is less complex, reliable and cost-effective. 


\section{Hardware and Software Description}

The hardware developed in this work includes CC3200-XL Launchpad, SW-520D tilt sensor and GSM SIM900A module. Energia Integrated Development Environment (IDE) is used to develop the firmware. The methodology includes sensing the tilt motion using SW-520D. CC3200 will collect the sensors data and uploads to the cloud using its on-chip Wi-Fi feature. The sensors real time data will be represented in the form of graphs. The hardware and description and software description are given below.

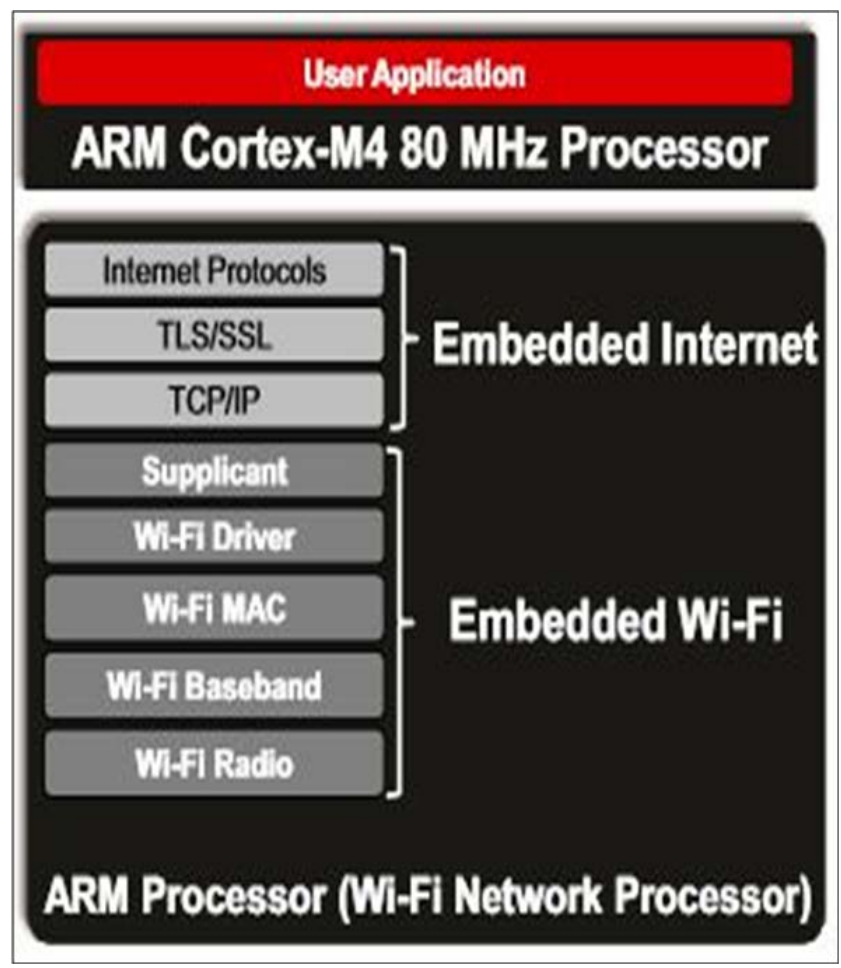

Figure 2. Overview of Wi-Fi stack architecture of CC3200 Launchpad.

\subsection{CC3200 XL-Launchpad}

CC3200 is the industry's first Wi-Fi certified on-chip from Texas instruments with an arm cortex core at $80 \mathrm{MHz}$. The board contains two on-chip sensors Temperature sensor (TMP006) and Accelerometer (BMA222) and having multiple protocols like UART, SPI, TCP/IP, I2C, TLS/ SSL status and HTTP server. For debugging purpose, the board consists of USB-JTAG header and for programming it has $8 \mathrm{MB}$ of flash memory. The board has an integrated DC-DC converter with voltage mode of 2.1 to $3.6 \mathrm{~V}$. It has a wide variety of peripherals like 4-ADC Channels, 20-pin headers, three LEDs. The feature of CC 3200 is IoT that includes $802.11 \mathrm{~b} / \mathrm{g} / \mathrm{n}$ protocol network processing system ${ }^{15}$. The Wi-Fi stack architecture over view of CC3200 is shown in the Figure 2.

\subsection{SW-520D Tilt Sensor}

Recent technical developments in the fabrication of tilt sensors leads to accuracy, cost-effective, reduced size and increased lifetime. The type SW-520D a 3-pin header, contains two poles acts like conductive elements and a conductive free mass acts like a rolling ball encapsulated in the same area and works with voltage range of 3.3$5 \mathrm{~V}$. The module looks tiny and is built around the dual comparator IC LM393, which acts as a signal amplifier. It consists of a power LED to check module power and the output LED to check whether the tilt is detected or not. The schematics of the Tilt sensor are shown in the Figure 3.

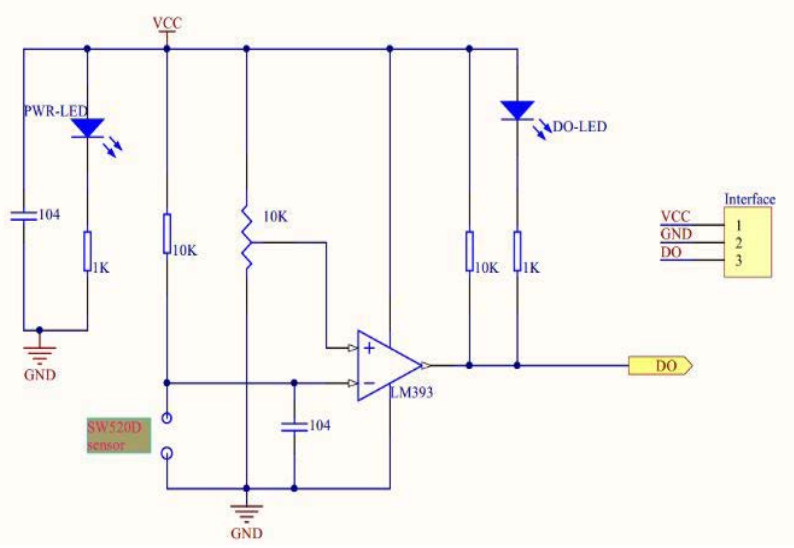

Figure 3. Schematics of the Tilt sensor.

When the sensor is in the upright position, the status of the output LED is $0 \mathrm{~V}$ (Low) and the ball inside bridges the two contacts and completes the circuit. When the sensor is tilted above 45 degrees the ball moves out so that the circuit opens and status of the output LED is $5 \mathrm{~V}$ (High) ${ }^{16}$.

\subsection{GSM SIM 900A MODULE}

Global System for Mobile (GSM) communication Subscriber Identity Module (SIM) 900A Module from SIMCOM. Its working frequency ranges from 900/1800 MHz. The baud rate is between 9600-115200. The modem requires only 2 wires (TX and RX) except power supply for interfacing. It consists of low drop linear voltage regulator used to connect wide range of unregulated 
power supply $(4.2-13 \mathrm{~V})$. It consists of SIM card holder, L type Antenna, network status LED and power LED. The powerful inbuilt TCP/IP protocol allows to Send and Read SMS through AT commands as shown in Table 1 and connected to the Internet through GPRS ${ }^{17}$.

Table 1. AT commands of the GSM Module used in the Present study

\begin{tabular}{ll}
\hline At Commands & Explanation \\
\hline AT & Attention \\
AT + CMGF & Set module in message mode \\
AT+CMGS & Set module in call mode \\
\hline
\end{tabular}

\subsection{Integration of Sensors with Microcontroller and Cloud}

In the present work, two tilt sensors are attached to the trunk position and the other to the back-rest position. The digital output of the first tilt sensor (Trunk position tilt sensor) is connected to the GPIO-12 (General-Purpose Input-Output) pin of CC3200 which is used to detect the trunk position of the patient lying on the wheel chair/ adjustable bed. The digital output of the second tilt sensor (back-rest tilt sensor) is connected to GPIO-13 pin of CC3200 which is used to detect the back-rest position of the patient lying on the adjustable bed/wheel chair as shown in Figure 4 and 5.

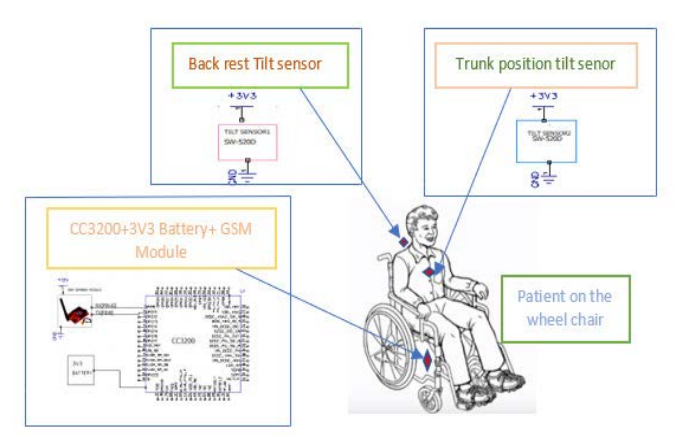

Figure 4. Hardware overview of the developed system for the patient on wheel chair.

CC3200 microcontroller will collect the data from these two sensors continuously and uploads to the Thing Speak cloud using its on-chip Wi-Fi feature. GSM module is connected to CC3200 through UART communication. The TX pin of GSM is connected GPIO-11 which is RX pin of CC3200 and RX pin of GSM is connected to GPIO10 which is TX pin of CC3200. If there is any abnormality detected in the patient's position then the GSM module will send an alert to the registered mobile number. The real time data can be viewed in PC/Laptop and the location of the patient will be displayed on the thing speak website. The entire schematics of the system are shown in Figure 6.

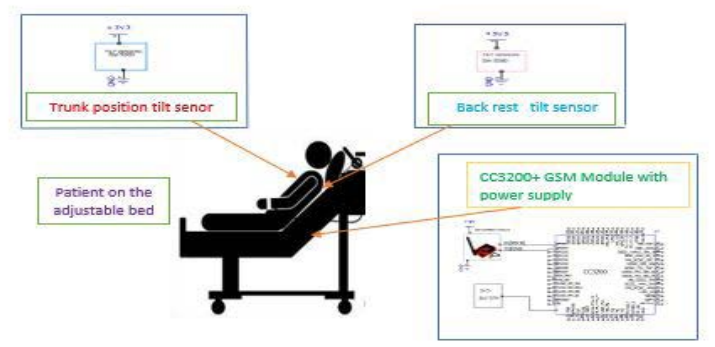

Figure 5. Hardware overview of the developed system for the patient on the adjustable bed.

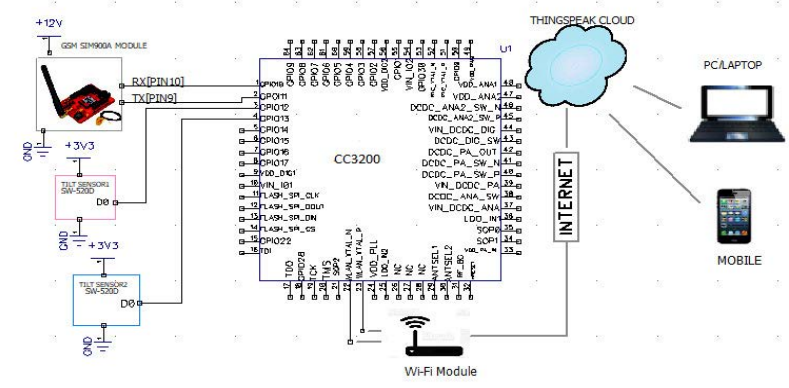

Figure 6. Schematics of the developed system.

\subsection{Thing Speak Cloud}

Thing Speak is an open data platform with MATLAB analytics. This cloud accesses the sensors data in real time and represents the data in the form of graphs and charts. The features of this cloud include analysing and visualizing the sensor's data using MATLAB. The applications involve in creating Plugins, Thing Tweet, React, Talk Back, Thing HTTP and Event Triggering ${ }^{18}$. The provision of this cloud is the thing view mobile application by which the sensors data can be viewed on Android mobiles. The method of accessing the cloud is explained:

1. The first step is to create a thing speak account at https://thingspeak.com. 
2. Sign in to the account and create new channel. Then the channel ID will be created automatically by the cloud which is unique for every channel. Every channel contains eight data fields for displaying data. Specify the name of the channel and also the channel as either Public or Private in the channel settings. The screen shot of channel setting window is shown in Figure 7.

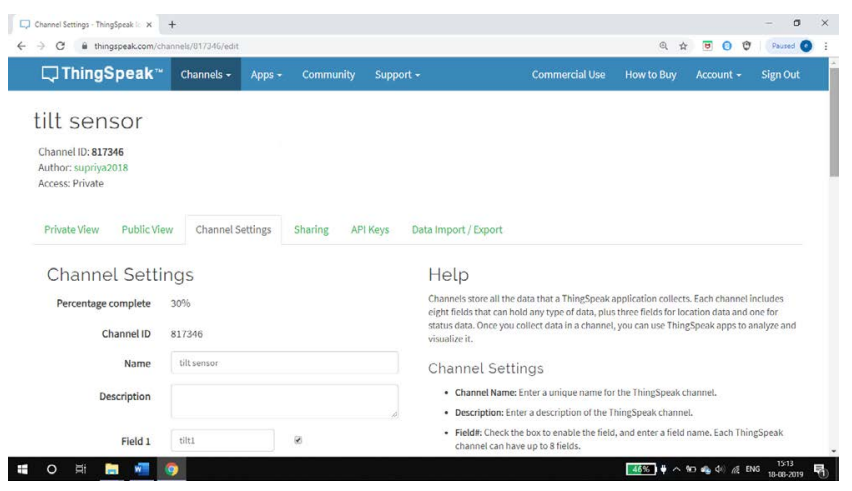

Figure 7. Screenshot of channel setting window.

1. After creating the channel with name, for accessing the data the cloud generates write and read API keys which are shown in Figure 8 as a screen shot.

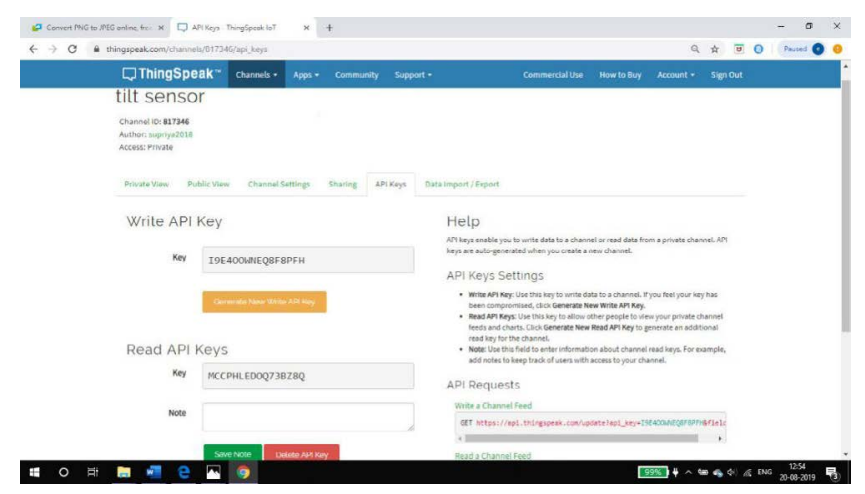

Figure 8. Screen shot of creating READ and WRITE API key.

\subsection{Software Description}

The firmware is developed by using Energia IDE in the present work. The flowchart for the developed work as shown in the Figure 9. Energia is an open source and community driven IDE and software framework. Energia supports various TI processors and is simple, easy-to-use code editor, and compiler with built in serial monitor/ terminal. It features a robust frame work of intuitive functional APIs for controlling a microcontroller ${ }^{19}$.

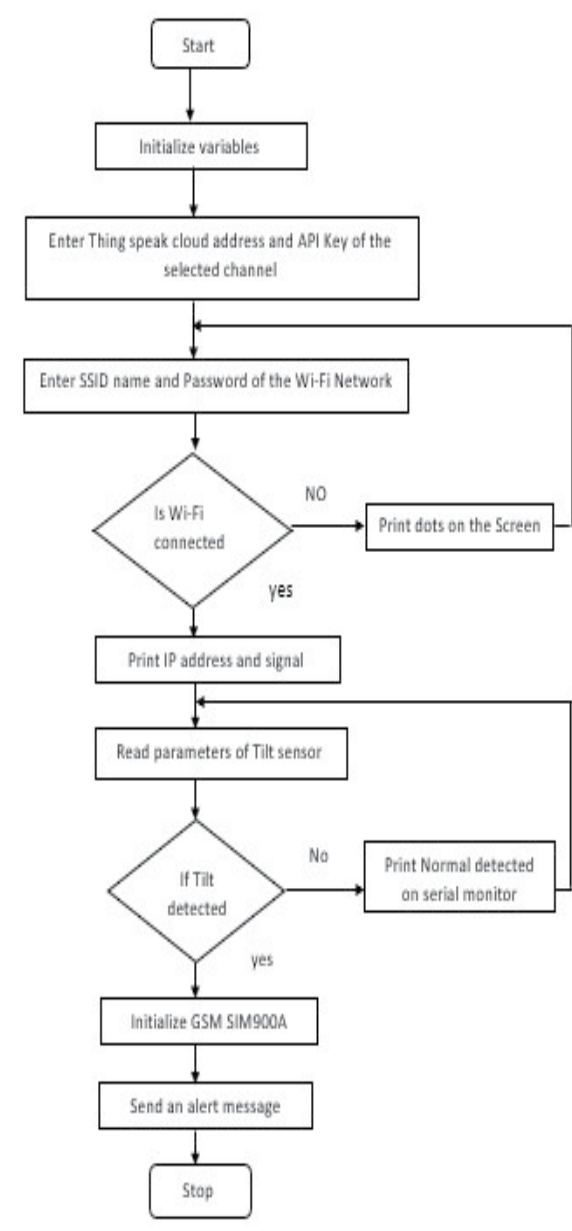

Figure 9. Flowchart of the program developed in the present work.

\section{Results and Discussion}

In this study we have developed a Patient fall detection system especially for the disable people/older adults using a low- cost sensor SW-520D. The patient titled position is simulated using the tilt sensor attached to the hand using a band. The working of the tilt sensor in four quadrants (of semicircle) tilted and normal postures of the hand are shown in Figure 10. CC3200 microcontroller is used to acquire the sensors data and uploaded the values to thing speak cloud and these values are stored in the cloud and the graphical representation of both sensors data in Field 1 and 2 is shown in Figure 11 and the location of the patient is tracked by using GeoTagging as shown in Figure 12. If the patient is tilted then GSM will send an alert to the mobile number and also the real time data of sensors can be downloaded from thing speak in the form of .CSV file as shown in Figure 13. 


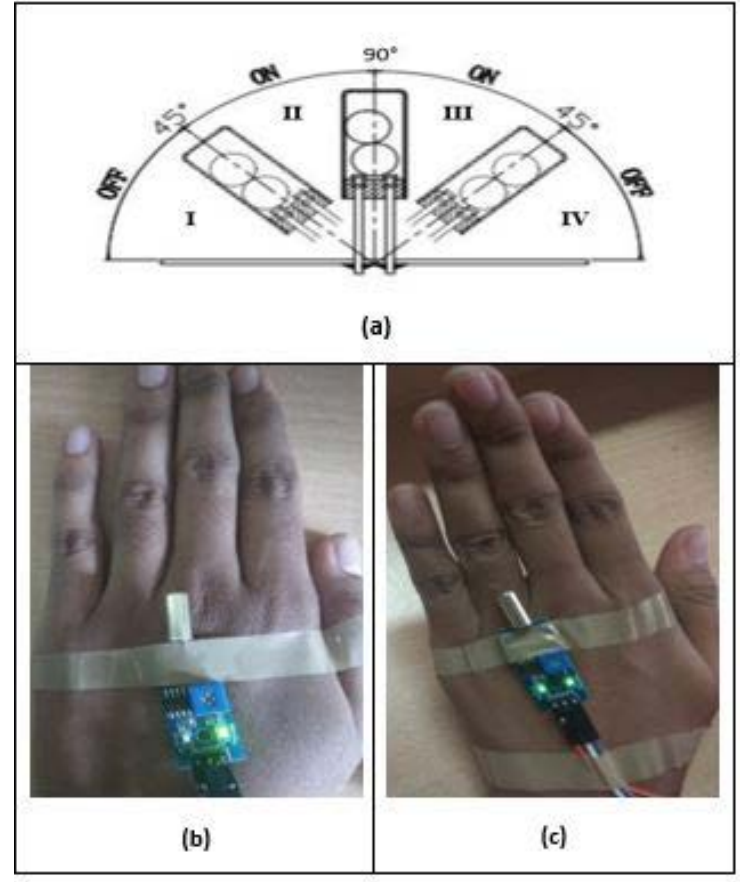

Figure 10. (a): Semi-circular four-quadrant working of the tilt sensor, (b): Hand posture is in quadrant I: power LED is HIGH, output LED is LOW, (c): Hand posture is in quadrant II: both power and output LEDs are HIGH.

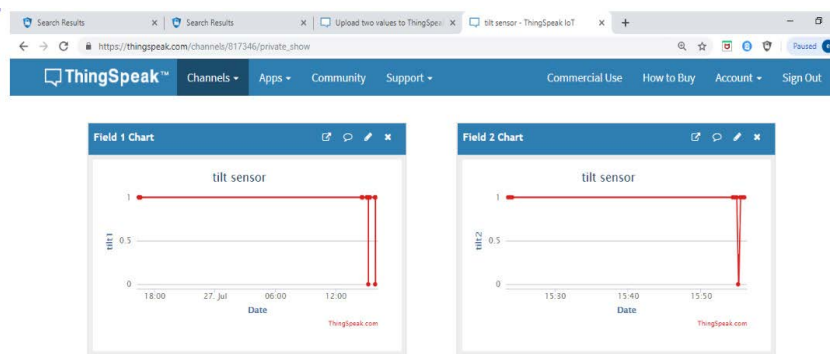

Figure 11. Screen shot of the real time data detected by 2- tilt sensors in field 1 and 2 .

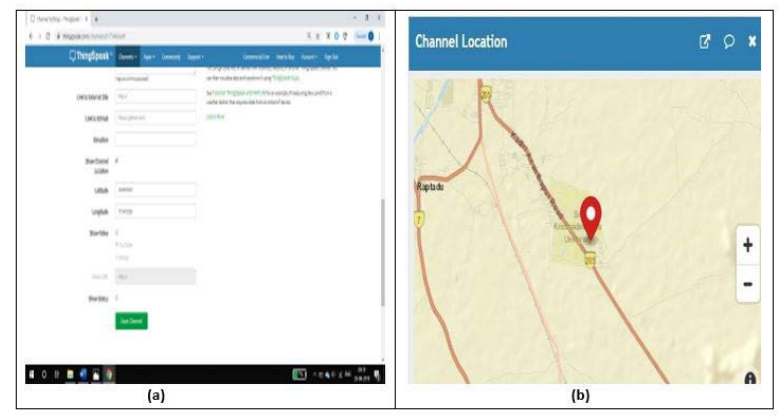

Figure 12. (a): Screenshots of the coordinates displayed on ThingSpeak website, (b): GeoTagging of sensors data with a location of Sri Krishnadevaraya University.

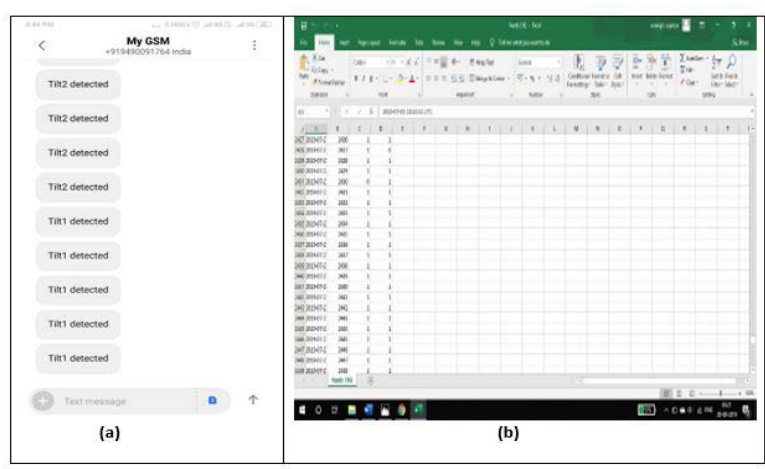

Figure 13. (a) Screenshot of the alert message sent to the mobile through GSM, (b): Sensors data retrieved in the form of excel sheet from cloud.

\section{Conclusion}

A low cost, continuous monitoring of Patient Fall Detection System [PFDS] with GeoTagging was developed. The system has a capability to continuously monitor the patient position using tilt sensor SW-520D and the sensors data are sent to the Thing Speak cloud for real-time observation and also an alert is sent to the nursing attendant and hospital dashboard using GSM module. The graphical representation of sensor values can be retrieved and viewed graphically anytime and anywhere in the world. The system has been composed of with minimum hardware components and with minimum power consumption by which system becomes cost-effective and robust.

\section{Acknowledgement}

K. Evangili Supriya is thankful to the Department of Science and Technology (DST), New Delhi, for sanctioning the INSPIRE fellowship and the authors are thankful to DST for sanctioning FIST program in establishing VLSI and Embedded Laboratory in the Department of Physics.

\section{References}

1. Cortes U, Urdiales C, Annicchiarico R, Barrue C, Martinez $\mathrm{AB}$, Cattagirine C. Assistive wheel chair navigation: A cognitive view, Studies in Computational Intelligence. 2017; 48:165-87. https://doi.org/10.1007/978-3-540-47527-9_7.

2. Pajkanovic A, Dokic B. Wheel chair control by head motion, Serbian Journal of Electrical Engineering. 2013; 10(1):13551. https://doi.org/10.2298/SJEE1301135P. 
3. Rechy E, Hu H, McDonald-Maier K. Head movementsbased control of an intelligent wheel chair in an indoor environment. 2012. IEEE International Conference on Robotics and Biometric [ROBIO]; 2012. p. 1464-69.

4. Davy M, Deepa R. Hardware implementation based on head movement using accelerometer sensor, International Journal of Applied Sciences and Engineering. 2014; 3(1):17-21.

5. Patel S, Park H, Bonato P, Chan L, Rodgers M. A review of wearable sensors and systems with application in rehabilitation, Journal of Neuroengineering and Rehabilitation. 2012; 9(21):1-17. https://doi.org/10.1186/1743-0003-9-21. PMid: 22520559 , PMCid: PMC3354997.

6. Kumar S, Dheeraj, Neeraj, Kumar S. Design and development of Head motion controlled wheel chair, International Journal of Advances in Engineering and Technology. 2012; $8(5): 816-22$.

7. Milekovic A, Otto C, Jovanov E. Wireless sensor networks for health monitoring: Issues and Implementation, Computer Communications. 2006; 29(13):2521-33. https://doi.org/10.1016/j.comcom.2006.02.011

8. Gupta A, Joshi N, Chaturvedi N, Sharma S, Pandar V. A Wheel chair control by head motion using accelerometer, International Journal of Electrical and Electronics Research. 2016; 4(1):158-61.

9. Vijayvargiya A, Yadav R, Sharma R, Meena R, Sharma S, Kanwar S, Siddiqui SA. Automatic wheel chair using accelerometer, International Journal of Electrical and Electronics Research. 2017; 5(2):5-7.

10. Debnath T, Abadin AFMZ, Hussain A. Android control smart wheel chair for disabilities, Global Journal of Computer Science and Technology. 2018; 18 (1):1-4.
11. Ding D, Leister E, Cooper RA, Cooper R, Kelleher A, Fitzgerald SG, Boninger ML. Usage of Tilt in space, recline and evaluation seating function in natural environment, Journal of Rehabilitation Research and Development. 2007; 42(7):973-84. https://doi.org/10.1682/JRRD.2007.11.0178. PMid: 19165687.

12. Nipanakar RS, Gaikwad V, Choudari C, Gosavi R, Vishal. Automatic wheel chair for physically disabled persons, International Journal of Advanced Research in Electronics and Communication Engineering. 2013; 2(4):466-74.

13. Shadwani M, Singh S, Verma VK, Sachan S. Intelligent solar based gesture controlled wireless wheelchair for the physically handicapped, Global Journal of Researchers in Engineering: Electrical and Electronics Engineering. 2016; 16(5):47-54.

14. Sundara Siva Kumar V, Ramesh G, Nagesh P. Mems based hand gesture wheel chair movement control for disable persons, International Journal of Current Engineering and Technology. 2015; 5(3):1774-76. https://doi.org/10.14741/ Ijcet/22774106/5.3.2015.48

15. CC3200 SimpleLink ${ }^{\mathrm{Tm}} \mathrm{Wi}^{-\mathrm{Fi}^{\oplus}}$ and Internet-of-Things Solution, a Single-Chip Wireless MCU. Date accessed: 2013. http://www.ti.com/lit/ds/symlink/cc3200.pdf.

16. SW-520D Tilt Sensor. Date accessed: 2019. https://www. sunrom.com/p/sw520d-sw-520d-tilt-sensor.

17. SIM900A GSM module datasheet. Date accessed: 2009. https://components101.com/sites/default/files/component_datasheet/SIM900A\%20Datasheet.pdf.

18. Thing Speak. Date accessed: 2019. https://thingspeak.com/.

19. Energia. Date accessed: 2019. https://energia.nu/. 